\title{
Déclaration de consensus du Réseau canadien des cliniques de SEP sur : l'utilisation des agents immunomodulateurs dans la sclérose en plaques
}

\author{
Paul O'Connor, Virginia Devonshire pour le Réseau canadien des cliniques de SEP
}

Can. J. Neurol. Sci. 2008; 35: 130-132

La déclaration suivante a trait aux médicaments actuellement approuvés par Santé Canada pour le traitement sclérose en plaques (SEP): Avonex ${ }^{\circledR}$ (Interféron Beta 1a), Betaseron ${ }^{\circledR}$ (Interféron Beta 1b), Rebif ${ }^{\circledR}$ (Interféron Beta 1a) Copaxone ${ }^{\circledR}$ (Acétate de Glatiramère) et Tysabri ${ }^{\circledR}$ (natalizumab). Nous recommandons, en outre, de prendre en considération la Novantrone $\circledR$ (mitoxantrone) dont l'utilisation dans la SEP n'est pas approuvée au Canada, mais l'est dans beaucoup d'autres pays comme traitement de la SEP.

Nous pensons cette déclaration nécessaire pour que tous les Canadiens atteints de SEP qui pourraient bénéficier de ces agents aient droit au traitement, même s'ils ne peuvent pas payer et ce, où qu'ils habitent au Canada.

Il existe des limitations à utiliser les critères choisis pour les essais thérapeutiques comme indicateurs d'un traitement clinique. Toutefois l'efficacité des thérapies immunomodulatrices n'a pas été établie en dehors des groupes traités lors des essais thérapeutiques. Par conséquent, ces directives reposent essentiellement sur les données des essais de phase 3 pour la SEP.

Chaque province devra mettre en place un mécanisme pour évaluer comment financer le traitement de tout/e patient/e dont le neurologue pense qu'il/elle bénéficierait d'une thérapie immunomodulatrice bien qu'il/elle ne réponde pas aux critères décrits ci-dessous.

\section{CRITÈRES PERMETTANT D'ENVISAGER UN \\ TRAITEMENT DANS LA SEP RÉMITTENTE CONFIRMÉE}

- Les patients doivent remplir les critères diagnostiques de la SEP, selon les critères revisés de McDonald. ${ }^{1}$

- L évolution de la maladie doit comprendre au moins une poussée clinique récente au cours de l'année précédant le traitement ou deux poussées dans les deux années précédentes. ${ }^{2-6}$

- Le patient doit être ambulatoire (si besoin avec aide).

- L'utilisation du natalizumab ne devrait normalement être envisagée que chez les patients dont la maladie continue à être nettement active (poussées ou IRM ) malgré le traitement par des agents de première ligne tel que l'interféron ou l'acétate de glatiramère.
Exceptionnellement, le natalizumab peut être utilisé comme agent de première ligne dans des cas de SEP agressive à rechutes où le risque paraît justifié.

\section{CRITÈRES PERMETTANT D'ENVISAGER UN TRAITEMENT DANS LES SYNDROMES CLINIQUEMENT ISOLÉS (SCI):}

Les patients doivent avoir eu un épisode neurologique documenté (p.ex. névrite optique, myélite ou syndrome du tronc cérébral) caractéristique de démyélinisation du système nerveux tel que ceux qu'on voit dans la SEP. Les syndromes cliniquement non localisables ne remplissent pas les conditions requises pour être qualifiés de SCI.

Une recherche exhaustive a été faite pour éliminer d'autres causes possibles de SCI et il n'y a pas de "meilleure explication" pour la présentation neurologique.

Les patients doivent être jugés comme courant un risque élevé d'avoir une autre poussée et donc de développer une SEP rémittente confirmée. La définition exacte de "risqué élevé" est encore sujet à discussion mais tous s'accordent à reconnaître que cela implique au moins la présence de multiples lésions T2 asymptomatiques sur l'IRM de l'encéphale avec ou sans rehaussement par le gadolinium au moment du SCI. ${ }^{8-10}$

La première poussée doit s'être produite durant l'année précédente.

A ce jour, les interférons beta-1a (Avonex et Rebif $22 \mathrm{mcg}$ ) et beta - $1 \mathrm{~b}$ (Betaseron) ont prouvé dans les essais thérapeutiques de phase 3 qu'ils retardaient la seconde poussée et donc la SEP cliniquement définie (SEPCD) ${ }^{8-10}$

\footnotetext{
From the Department of Neurology, St. Michael's Hospital (POC), Toronto, ON; University of British Columbia (VD), Vancouver, BC, Canada.

Received July 16, 2007. Final Revisions Submitted November 19, 2007. Reprint requests to: Paul O'Connor, Department of Neurology, St. Michael's Hospital, 30 Bond Street, Toronto, Ontario, M5B 1W8, Canada.
} 
CRITÈRES PERMETTANT D'ENVISAGER UN TRAITEMENT PAR LA MITOXANTRONE DANS LA SEP RÉMITTENTECONFIRMÉE :

Certains patients atteints de SEP ont une maladie particulièrement aggressive avec des poussées fréquentes sans rémission ou avec des rémissions incomplètes qui sont associées à une augmentation rapide de leur invalidité. Ces patients auront généralement essayé un traitement avec des agents immunomodulateurs de première ligne (interféron-beta ou acétate de glatiramère) ou peut-être le natalizumab sans qu'on puisse stabiliser la maladie de manière adéquate.

Dans cette situation le traitement à la mitoxantrone peut être mis en route pour réduire l'activité de la maladie et ralentir la progression de l'invalidité, mais cet agent, ainsi que d'autres immunosuppresseurs à large spectre, présente des risques de toxicité nettement plus élevés. ${ }^{11}$ La mitoxantrone peut provoquer une cardiomyopathie et favoriser le développement d'affections malignes (p.ex. leucémie). ${ }^{12}$

- Les patients doivent présenter une maladie très active, ceci généralement malgré l'utilisation d'autres thérapies immunomodulatrices, selon l'un ou l'autre des exemples suivants:

Rechutes multiples ( 2 ou plus) au cours de l'année précédente atteignant les systèmes moteur ou cérebelleux avec rémission incomplète et avec progression confirmée de l'incapacité.

\section{OU}

Progression rapide de l'incapacité clinique et preuve à l'IRM d'une inflammation active importante (p.ex. multiples lésions avec rehaussement).

- Il est nécessaire que le neurologue traitant ait une expertise particulière avec l'utilisation de la mitoxantrone et que le patient traité à la mitoxantrone soit suivi dans une clinique de SEP multidisciplinaire. Ceci est nécessaire, à la lumière de la toxicité cardiaque et hématologique qui a été documentée à la suite des traitements à la mitoxantrone.

- Le traitement doit être individualisé pour chaque patient et doit tendre à utiliser la plus petite dose efficace possible. L'innocuité d'immunothérapies comme le mitoxantrone après l'administration de natalizumab n'a pas encore été démontrée.

\section{CONDITIONS SUPPLEMENTAIRES S'APPLIQUANT A L'UTILISATION DE LA THERAPIE IMMUNOMODULATRICE}

- Les patients doivent accepter une surveillance régulière par le médecin prescripteur.

- Les patientes doivent accepter d'utiliser une méthode contraceptive appropriée.

- Un système doit être établi pour assurer l'éducation adéquate des patients avant le début du traitement, pour vérifier que les patients se conforment au traitement et pour surveiller les effets secondaires.

- Bien que ces traitements semblent retarder la progression à court terme, leur efficacité à long terme n'a pas été prouvée.

\section{IL EST ENCORE RECOMMANDE QUE:}

- Tous les patients remplissant les conditions requises soient considérés comme candidats pour le traitement à un stade précoce de leur maladie car il semble que les thérapies immunomodulatrices sont d'autant plus efficaces qu'elles sont utilisées tôt..$^{8-10}$

- Un bilan périodique des patients traités doit être fait pour évaluer efficacité et effets secondaires de manière à décider s'il est approprié de poursuivre le traitement.

- Étant donné que des taux élevés d'anticorps neutralisant dirigés contre l'interféron réduisent probablement son efficacité, il est recommandé de faire une évaluation des anticorps au bout d'au moins une année de traitement, surtout chez les patients dont la maladie est active..$^{13}$ La mesure de ces anticorps devrait être facile à obtenir car elle fait partie des soins normaux aux patients atteints de SEP.

- Il est reconnu que les problèmes qui découlent de l'utilisation des thérapies immunomodulatrices pour la SEP sont complexes et dépassent le cadre habituel de la pratique neurologique. Nous recommandons par conséquent que ces thérapies soient prescrites et surveillées par des neurologues ayant une expertise particulière dans les soins aux patients atteints de SEP.

\section{CONSIDERATIONS SPECIALES POUR LE NATALIZUMAB:}

- Le risque de contracter une leucoencéphalite multifocale progressive (LMP) reste un problème important pour tous les patients. Bien qu'une LMP soit apparue chez deux patients traités par une association d'interféron beta 1a (Avonex $\left.{ }^{\circledR}\right)$ et de natalizumab (Tysabri ${ }^{\circledR}$ ), on ne sait pas encore si c'est le résultat de la thérapie combinée ou si le même risque est associé au natalizumab en monothérapie. De plus, à ce jour, le natalizumab n'a été utilisé que jusqu'à 36 mois et on ignore quel est le risque de LMP et d'autres infections opportunistes s'il est utilisé plus longtemps.

- Il n'y a pas de directive établie sur la période de lavage thérapeutique quand on remplace un interféron ou l'acétate de glatiramère par un autre immunomodulateur. Cependant aucune manifestation négative n'est apparue quand un interféron beta et le natalizumab ont été pris en association pendant une durée de 18 mois. Un traitement antérieur à la mitoxantrone ou cyclophosphamide exigera une période de lavage thérapeutique. La durée nécessaire est incertaine et dépendra de la dose et de la durée de la chimiothérapie. La décision devra être individualisée.

- Une IRM devra être faite dans les trois mois qui suivent le début du traitement au natalizumab à des fins de comparaison, dans le but d'anticiper l'apparition d'une LMP.

- Tous les patients devront être suivis de près et de manière régulière sur le plan clinique pour surveiller d'éventuels signes et symptômes de LMP. Les patients devront être vus au minimum tous les 6 mois. La valeur de l'IRM de routine pour dépister la LMP est mal définie. La monographie du produit contient un algorithme à utiliser quand une LMP est soupçonnée. On ne sait pas si la recherche du virus JC dans le sérum a une valeur diagnostique et si elle peut servir de mesure de dépistage préventif.

- L'apparition d'anticorps neutralisants contre le natalizumab réduit son efficacité et provoque une augmentation des réactions lors de l'injection. La recherche d'anticorps doit être facile à obtenir et des tests doivent être envisagés si des 
réactions à l'injection se produisent ou si l'efficacité du médicament est mise en doute. L'apparition des anticorps se fait typiquement au bout de deux à six mois.

\section{CONSIDERATIONS POUR INTERROMPRE LE TRAITEMENT IMMUNOMODULATEUR}

- Les traitements doivent être interrompus (et non changés) chez les personnes qui n'ont pratiquement pas de chance d'en bénéficier à cause du niveau avancé de leur incapacité (p. ex. non ambulatoires)

- Il est admis que la SEP évolue graduellement d'une phase précoce où l'inflammation est dominante au cours de laquelle les traitements actuels peuvent maximiser leur effet bénéfique, à une phase plus dégénérative qui ne semble pas être affectée par ces agents anti-inflammatoires. La transition entre ces deux phases est graduelle et caractérisée par une diminution des attaques cliniques et de la prise de Gadolinium à l'IRM en même temps qu'une augmentation lente de l'incapacité physique. L'interruption du traitement doit donc être envisagée chez les patients qui n'ont pas eu de poussée depuis plusieurs années et dont l'incapacité continue à augmenter.

Cette déclaration de consensus a été préparée par les Drs $\mathrm{P}$. O'Connor et V. Devonshire avec la contribution et l'approbation des membres du Réseau canadien des cliniques de SEP dont les noms suivent:

Antel J, Arnold D, Bhan V, Bar-Or A, Bakker J, Bell R, Boyle C, Brunet D, Costello F, Duquette P, Freedman M, Gomori A, Grand-Maison F, Gray TA, Hader W, Hohol M, Hrebicek O, Hooge J, Kremenchutzky M, Lee L, Marrie RA, Murray J, Myles ML, Metz L, Oger J, Patry DG, Prat A, Rabinovitch H, Short C, Smyth P, Traboulsee A, Vorobeychik G.

\section{RÉFÉRENCES}

1. Polman CH, Reingold SC, Edan G, Filippi M, Hartung HP, Kappos L, et al. Diagnostic criteria for multiple sclerosis: 2005 revisions to the McDonald Criteria. Ann Neurol. 2005; 58(6):840-6.

2. IFNB Multiple Sclerosis Study Group. Interferon beta- $1 \mathrm{~b}$ is effective in relapsing-remitting multiple sclerosis. I. Clinical results of a multicenter, randomized, double-blind, placebocontrolled trial. Neurology. 1993; 43:655-61.
3. Jacobs LD, Cookfair DL, Rudick RA, Herndon RM, Richert JR, Salazar AM, et al. Intramuscular interferon beta-1a for disease progression in relapsing multiple sclerosis. Ann Neurol. 1996; 39:285-94.

4. PRISMS Study Group. Randomized double-blind placebocontrolled study of interferon [beta]-1a in relapsing/remitting multiple sclerosis. Lancet. 1998; 352:1498-504.

5. Johnson K, Brooks B, Cohen JA, Ford CC, Goldstein R, Lisak RP, et al. Copolymer 1 reduces relapse rate and improves disability in relapsing-remitting multiple sclerosis: results of a phase III multicenter, double-blind, placebo-controlled trial. Neurology. 1995; 45:1268-76.

6. Polman $\mathrm{CH}, \mathrm{O}^{\prime}$ Connor PW, Haverdova E, Hutchinson M, Kappos $\mathrm{L}$, Miller DH, et al. A randomized, placebo-controlled trial of natalizumab for relapsing multiple sclerosis. N Engl J Med. 2006; Mar 2;354(9):899-910.

7. Health Canada-Product Monograph 2006.

8. Jacobs LD, Beck RW, Simon JH, Kinkel RP, Brownscheidle CM, Murray $\mathrm{TJ}$, et al. Intramuscular interferon beta-1a therapy initiated during a first demyelinating event in multiple sclerosis. N Engl J Med. 2000; 343:898-904.

9. Comi G, Filippi M, Barkhof F, Durelli L, Edan G, Fernandez O, et al. Effect of early interferon treatment on conversion to definite multiple sclerosis: a randomized study. Lancet. 2001; 357: 1576-82.

10. Kappos L, Polman CH, Freedman MS, Edan G, Hartung HP, Miller $\mathrm{DH}$, et al. Treatment with interferon beta- $1 \mathrm{~b}$ delays conversion to clinically definite and McDonald MS in patients with clinically isolated syndromes. Neurology. 2006; 10;67(7):1242-9.

11. Hartung HP, Gonsette R, Konig N, Kwiecinski H, Guseo A, Morrissey SP, et al. Mitoxantrone in progressive multiple sclerosis: a placebo-controlled, double-blind, randomized, multicentre trial. Lancet. 2002; 360(9350):2018-25.

12. Goodin DS, Arnason BG, Coyle PK, Frohman EM, Paty DW. Therapeutics and Technology Assessment Subcommittee of the American Academy of Neurology. The use of mitoxantrone (Novantrone) for the treatment of multiple sclerosis: report of the Therapeutics and Technology Assessment Subcommittee of the American Academy of Neurology. Neurology. 2003; 61(10): $1332-8$.

13. Goodin DS, Frohman EM, Hurwitz B, O'Connor PW, Oger JJ, Reder AT, et al. Neutralizing antibodies to interferon beta: assessment of their clinical and radiographic impact: an evidence report: report of the Therapeutics and Technology Assessment Subcommittee of the American Academy of Neurology. Neurology. 2007; 68(13):977-84. 